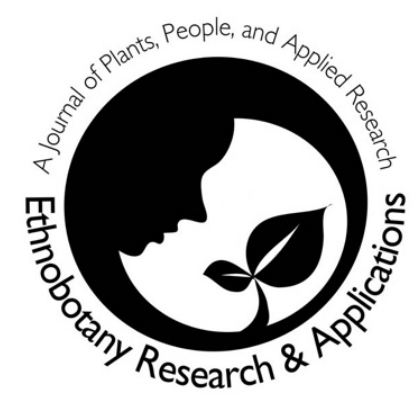

\title{
Senecio tephrosioides Turcz. (Asteraceae): Una revisión de etnobotánica, fitoquímica y farmacología
}

\author{
Cyntia Blanco-Olano, Karyn Olascuaga-Castillo, Susana \\ Rubio-Guevara, Juan E. Valdiviezo-Campos
}

\section{Mini Review}

\begin{abstract}
Resumen
Antecedentes: Senecio tephrosioides Turcz. (Familia: Asteraceae) es una planta medicinal tradicional utilizada en varios sistemas de medicina antigua en el Perú por su valor etnofarmacológico para curar variedades de dolencias. Los terpenos (1isopropil- 4-metil biciclo $[3,1,0]$ hexano y terpineno), compuestos fenólicos (5,7-dihidroxi-4',6',8trimetoxiflavanonona, 3',5'-dihidroxi-4-metoxi-7-0-

rhannoglucosyl flavanonona y 5,4dihidroxichalcona), flavonoides (quercetin-3rutinósido), alcaloides de pirrolizidina (neoplatifilina, Senecionina, seneciphylline, seneciphylline N-óxido y senkirkine), y lactonas, son los principales componentes activos obtenidos de las partes aéreas de este género, y los cuales guardan relación importante en preparaciones tradicionales para el tratamiento y manejo de enfermedades. El presente mini-review se centra principalmente en los aspectos botánicos, la etnofarmacología, los principales metabolitos secundarios, y los posibles usos medicinales de Senecio tephrosioides.
\end{abstract}

Materiales y Métodos: Las fuentes para la búsqueda de literatura fueron Scielo, PubMed, ScienceDirect and Google Scholar.

Resultados: Las actividades farmacológicas informadas, los metabolitos secundarios y los usos tradicionales de Senecio tephrosioides justifican sus posibles usos terapéuticos.

Conclusions: Sin embargo, aún se requieren más estudios que vayan de la mano con validaciones científicas y estandarizaciones utilizando métodos ortogonales como Resonancia Magnética Nuclear (RMN), Cromatografía Liquida de Alta Resolución
(HPLC) y Espectrometría de Masas - acoplado a Cromatografía Liquida (MS-LC), contrastar el conocimiento tradicional con evidencia a través de ensayos metodológicos que permitan determinar la seguridad y funcionamiento de los componentes de esta especie, así como una oportunidad en el descubrimiento y desarrollo de nuevos medicamentos a partir de productos naturales purificados o extractos crudos particionados.

Palabras clave: Senecio tephrosioides; Huamanripa; Huamanripa; wamanripa; usos tradicionales; Planta medicinal

\section{Correspondence}

Cyntia Blanco-Olano*, Karyn Olascuaga-Castillo, Susana Rubio-Guevara, Juan E. ValdiviezoCampos

Facultad Farmacia y Bioquímica, Universidad Nacional de Trujillo. Av. Juan Pablo II s/n. 13011. Trujillo-Perú

“Corresponding Author cblanco@unitru.edu.pe

\section{Ethnobotany Research \& Applications} 19:14 (2020)

\section{Antecedentes}

Las plantas cumplen un papel fundamental en la historia del hombre, ya que desde la antigüedad han sido un recurso muy importante en diversas necesidades en la vida del ser humano; sin embargo, el empleo de plantas en la medicina no ha perdido el interés, aun en la actualidad cientos de plantas son utilizadas en diferentes campos como, industria alimentaria, industria cosmética, industria farmacéutica, etc. Así mismo, es importante mencionar la utilidad de plantas en el desarrollo de las culturas andinas, ya que desde la llegada del 
hombre a los andes, estos utilizaban los recursos vegetales como fuente de alimento, medicina, combustible, materiales de construcción y herramientas de todo tipo; las plantas han ocupado incluso un lugar importante en su sistema de creencias y ritos (De la Torre et al. 2006), en la actualidad, los principales usos que se le está dando a las plantas a nivel mundial, es en el campo de la medicina, particularmente, el uso extensivo de las plantas medicinales en los andes es una tradición que continúa hasta la fecha, donde el conocimiento ha ido transmitiéndose de generación en generación (Bussmann et al. 2007).

La Medicina Tradicional y la Medicina AlternativaComplementaria están ganando más y más respeto de gobiernos nacionales (Bussmann \& Sharon 2015). En América Latina, la Oficina Regional de la Organización Mundial de Salud de las Américas (OMS/OPS) reporta que alrededor del $70 \%$ de la población ha usado la Medicina Tradicional. En Perú, la medicina tradicional se ha articulado a la medicina complementaria (MC), definida como un amplio conjunto de prácticas de atención de salud que no forman parte de la tradición ni de la medicina convencional de un país dado ni están totalmente integradas en el sistema de salud predominante. Las tendencias de uso de plantas medicinales en el país indican que casi el $80 \%$ de la población conoce el uso de la fitoterapia como recurso medicinal (Mejía et al. 2017). Según el Instituto Cuánto y la ONG Prisma, se ha verificado que el $76 \%$ de los asegurados de EsSalud están dispuestos a recibir tratamiento con plantas medicinales (OPS 2019), mientras que cerca de 90000 asegurados por año utilizan los servicios de medicina complementaria, enmarcados en los 83 centros de atención de Medicina Complementaria que existen en Perú (SES 2017). Sin embargo, se requiere dar mayor énfasis en el control de calidad de plantas medicinales, ya que estos mismos pueden verse afectados por diversos factores, por ejemplo, la práctica de recolección degradante que se ve afectada por la alta variabilidad en la calidad de la materia prima, generando productos que no están estandarizados y que presentan diferentes efectos en la salud. Como consecuencia, se pierde la credibilidad en el mercado de la MC, evidenciando la necesidad de promover la investigación en este campo.

El género Senecio está formado por más de 2000 especies con una amplia distribución mundial (da Silva et al. 2006), 97 de las cuales se encuentran en Perú (Beltran 2016). Senecio tephrosioides es una especie popular utilizada como planta medicinal medicinal y se distribuye principalmente al sur de Perú; su uso y distribución también se encuentra en Brasil, Argentina, Paraguay y Uruguay (da Silva et al. 2006). Senecio tephrosioides se conoce bajo el nombre vulgar de huamanripa en la medicina tradicional peruana, esta planta medicinal es aplicad para tratar las siguientes enfermedades: resfriados, infección del tracto respiratorio superior, neumonía, bronquitis, asma, diaforesis, expectorante (Bussmann \& Sharon 2015, Mostacero et al. 2011). En Perú se han realizado pocos estudios fitoquímicos sobre las diferentes especies de Senecio, cosa que llama la atención debido a sus diversos usos medicinales tradicionales. Los compuestos químicos encontrados en esta planta son: terpenos (1-isopropil- 4-metil biciclo [3,1,0] hexano y terpineno), compuestos fenólicos $(5,7-$ dihidroxi-4',6', 8-trimetoxiflavanonona, 3',5'-dihidroxi4-metoxi-7-0-rhannoglucosyl flavanonona y 5,4dihidroxichalcona), flavonoides (quercetin-3rutinósido), alcaloides de pirrolizidina (neoplatifilina, Senecionina, seneciphylline, seneciphylline N-óxido y senkirkine), y lactonas, los cuales requieren ser estudiados a mayor profundidad. Ya que se ha encontrado información en diversos estudios que diversas especies del género Senecio incluyen alcaloides de pirrolizidina (AP) (Bussmann et al. 2011, Habermehl et al. 1988), que son conocidos como hepatotoxinas y carcinógenos (da Silva et al 2006). Teniendo en cuenta el riesgo que implica la exposición humana a los AP, la Organización Mundial de la Salud (OMS) emitió una Guía de salud y seguridad en 1989 sobre el uso de plantas medicinales que contienen dichos compuestos. En consecuencia, el uso de plantas medicinales Senecio se ha restringido en algunos países occidentales desarrollados. En este estudio se revisan la botánica, la etnofarmacología, la fitoquímica y los posibles usos medicinales de Senecio tephrosioides. También se evalúan los métodos eficientes utilizados para las técnicas analíticas de Senecio y también se analizan los estudios de toxicidad, que son esenciales para la evaluación de las diversas especies de Senecio, una opción clínica segura.

\section{Material y Métodos}

La búsqueda de información para la elaboración de esta revisión fue realizada en los motores de búsqueda y base de datos Scielo, PubMed, ScienceDirect and Google Scholar, con las siguientes palabras clave: "Senecio tephrosioides", "Huamanripa", "Huamanripa", "wamanripa", "Asteraceae", "usos tradicionales", "Planta medicinal", "biological activity", "traditional use", y se utilizó los operadores booleanos 'AND'/ 'OR'. Los criterios de inclusión de la información fueron artículos originales y de revisión, tesis de grado y postgrado, no se descartaron resultados por año de publicación, los idiomas se redujeron a español e inglés. En el análisis de la información se revisaron 
los resúmenes y de contener información relevante se consultó el artículo completo, verificando en primer lugar que los trabajos muestren un registro de ingreso o que cuenten con un certificado de caracterización taxonómica emitida por herbarios nacionales (Herbarium Truxillense (HUT), Herbario Museo de Historia Natural (MHN), Herbario Weberbauer de la Universidad Nacional Agraria La Molina (MOL), Herbario Vargas Cuz (CUZ).

\section{Resultados y Discusión}

La búsqueda en las bases de datos descritas anteriormente, tuvo como resultado para el género Senecio 2575 resultados entre artículos y tesis relacionadas a esta revisión, de estos 138 resultados corresponden a Senecio tephrosioides luego de eliminar los resultados repetidos y ordenar por relavancia se obtuvieron 44 artículos. Las investigaciones relevantes acerca de Senecio se describen en la Tabla 1.

\section{Senecio: uno de los géneros cosmopolitas más exitosos}

Se ha estimado que Perú tiene 17,143 taxa de espermatofitas en 2485 eco-regiones y 224 familias con $7 \%$ de las plantas del mundo (Brako \& Zaruchi 1993), a muchas de estas plantas se le atribuyen ciertas propiedades terapéuticas, de allí, la importancia de identificar correctamente y estudiar a cada una de estas plantas, ya que, reconociendo sus características, permite que exista una base científica. De allí, las Astereaceas ocupan el segundo lugar entre las familias más diversas de la flora peruana (Beltrán et al. 2006).

Uno de los géneros con una mayor cantidad de especies perteneciente a esta familia es el género Senecio (Brako \& Zarucchi 1993). El género Senecio fue descripto por Linné en el año 1753 en su obra Species Plantarum. Debe su nombre al vocablo latino senex que significa anciano (Milton 2009), es un género muy variable en cuanto al número de especies que lo componen, incluyen alrededor de 250 especies con un amplio espectro de formas de vida que se encuentran en todo el mundo y se caracteriza por tener actividad en el tratamiento de asma bronquial y también se han reportado resultados óptimos de efecto antinflamatorio, antimicrobiano, analgésico y antioxidante (Beltrán 2006).

Sin embargo, este género exhibe importantes conflictos debido a que sus límites no están claramente definidos y la identificación de las especies es dificultosa (Cabrera 1957; López 2008). Aun así, Senecio es uno de los géneros cosmopolitas más exitosos; debido a que se encuentran prácticamente en todo tipo de hábitat, haciéndose más diverso en las regiones montañosas templadas y frías del mundo (Rojas 2001). Está distribuido por todo el mundo a excepción de las islas del Pacífico y del continente Antártico. La mayor concentración de especies se encuentra en las regiones montañosas de América, Asia y África (Cabrera et al. 1999).

Perú cuenta con una cantidad resaltante del genero Senecio (López et al. 2013), es considerado como el género más distribuido y amplio de todas las Asteráceas con interés ecológico y económico. Las especies presentes en el Perú muestran gran diversidad y una distribución que abarca diferentes altitudes entre la costa y la sierra en diferentes nichos ecológicos, alcanzando su mayor variabilidad en hábitats andinos; variando desde hierbas anuales a perennes rizomatosas, arbustos sufrutescentes o arbustos. Las hojas son basales, caulinares, o ambos tipos (Vision \& Dillon 1996).

Beltrán et al. (2006) categorizaron las especies peruanas de acuerdo a su estado de conservación (IUCN Standards and Petitions Subcommittee 2014), en donde indica que 100 especies de Senecio son endémicas para Perú. Sin embargo, los estudios moleculares aún son escasos para el género Senecio (Nordenstam et al. 2009) y sus relaciones filogenéticas no son aún concluyentes (Pelser et al. 2007).

Las especies de Senecio se hallan en la costa y sierra del Perú, donde son denominadas comúnmente bajo el mismo nombre vulgar de "Huamanrripa" o "Huira-huira" y comparten características morfológicas similares (Senecio calvus, S. tephrosioides, $S$. comosus, $S$. rhizomattus, S. klatti, etc). Las especies de este género en nuestro país representan aproximadamente el $60 \%$ de la diversidad de Senecio en Sudamérica (Florián 2014).

Su aplicación en la medicina tradicional, está relacionado principalmente como antitusígeno y para procesos respiratorios en forma de infusión, en general las plantas de este género son utilizadas también como antiiflamatorios, antimicrobianos y analgésicos (Deuschle et al. 2006). Entre las especies de Senecio que se han descrito como utilizadas en la medicina tradicional se hallan Senecio tephrosioides, S. canescens, $S$. chionogeton, S. mathewsii, S. rudbeckiaefolius (Florián 2014). 
Tabla 1. Resumen de los estudios acerca del género Senecio

\begin{tabular}{|c|c|c|c|c|c|c|c|}
\hline Especie & $\begin{array}{l}\text { Nombre } \\
\text { vulgar }\end{array}$ & Procedencia & Uso tradicional & Compuestos & $\begin{array}{l}\text { Forma de } \\
\text { preparación }\end{array}$ & $\begin{array}{l}\text { Parte } \\
\text { utilizada }\end{array}$ & Autor \\
\hline $\begin{array}{l}\text { Senecio } \\
\text { tephrosioides } \\
\text { Turcz. }\end{array}$ & "Huamanripa" & $\begin{array}{l}\text { Callejón de } \\
\text { Huaylas }\end{array}$ & Tos y bronquitis & & Decocción & Hoja & $\begin{array}{l}\text { Hammond } \\
\text { et al. } 1998\end{array}$ \\
\hline $\begin{array}{l}\text { Senecio culcitioides } \\
\text { Schultz-Bip }\end{array}$ & "Asnac-ccora" & $\begin{array}{l}\text { Callejón de } \\
\text { Huaylas }\end{array}$ & $\begin{array}{l}\text { Tos, bronquitis y } \\
\text { asma }\end{array}$ & & Decocción & Hoja & $\begin{array}{l}\text { Hammond } \\
\text { et al. } 1998\end{array}$ \\
\hline $\begin{array}{l}\text { Senecio } \\
\text { rhizomatosus } \\
\text { Rusby }\end{array}$ & "Llanchuasa" & $\begin{array}{l}\text { Callejón de } \\
\text { Huaylas }\end{array}$ & $\begin{array}{l}\text { Curación de } \\
\text { heridas, aumentar } \\
\text { secreción biliar }\end{array}$ & & Infusión & Hoja & $\begin{array}{l}\text { Hammond } \\
\text { et al. } 1998\end{array}$ \\
\hline $\begin{array}{l}\text { Senecio } \\
\text { culcitoides Weed }\end{array}$ & $\begin{array}{l}\text { "huira-huira", } \\
\text { "huajchor" }\end{array}$ & Huarochirí, Lima & $\begin{array}{l}\text { Cicatrizante, } \\
\text { antinflamatorio }\end{array}$ & $\begin{array}{l}\text { quercetin-3-rutinósido; } \\
\text { 5,7-dihidroxi-4',6',8- } \\
\text { trimetoxiflavanonona,3',5'- } \\
\text { dihidroxi-4-metoxi-7-0- } \\
\text { rhannoglucosyl } \\
\text { flavanonona; } \\
\text { 5,4-dihidroxichalcona }\end{array}$ & $\begin{array}{l}\text { Infusión - } \\
\text { Maceración }\end{array}$ & Hoja & $\begin{array}{l}\text { Soriano et } \\
\text { al. } 2004\end{array}$ \\
\hline $\begin{array}{l}\text { Senecio } \\
\text { tephrosioides } \\
\text { Turcz. }\end{array}$ & $\begin{array}{l}\text { "Huamanripa", } \\
\text { "huamanripa } \\
\text { verdadera", } \\
\text { "huamanlipa", } \\
\text { "huaman } \\
\text { hembra" }\end{array}$ & $\begin{array}{l}\text { Piura, Ancash, } \\
\text { Cusco, Puno }\end{array}$ & $\begin{array}{l}\text { Diaforética, } \\
\text { enfermedades de } \\
\text { vías respiratorias, } \\
\text { expectorante, } \\
\text { gripe }\end{array}$ & & Infusión & Hojas & $\begin{array}{l}\text { Mostacero } \\
\text { et al. } 2011\end{array}$ \\
\hline $\begin{array}{l}\text { Senecio canescens } \\
\text { (H.B.K.) } \\
\text { Cuatrecasas }\end{array}$ & $\begin{array}{l}\text { "Vira Vira", } \\
\text { "Oreja de } \\
\text { Conejo", }\end{array}$ & Andes, Perú & $\begin{array}{l}\text { Bronquitis, Asma, } \\
\text { Tos, Nervios }\end{array}$ & & Decocción & $\begin{array}{l}\text { Planta } \\
\text { entera }\end{array}$ & $\begin{array}{l}\text { Bussmann } \\
\text { \& Sharon } \\
2015\end{array}$ \\
\hline $\begin{array}{l}\text { Senecio comosus } \\
\text { Sch.-Bip. }\end{array}$ & $\begin{array}{l}\text { "Hórnamo", } \\
\text { "Leon } \\
\text { Amarillo" }\end{array}$ & Andes, Perú & $\begin{array}{l}\text { Mal Aire, } \\
\text { Inflamación } \\
\text { (general), } \\
\text { Mejoramiento de } \\
\text { visiones }\end{array}$ & & Decocción & $\begin{array}{l}\text { Hojas, } \\
\text { tallo }\end{array}$ & $\begin{array}{l}\text { Bussmann } \\
\text { \& Sharon } \\
2015\end{array}$ \\
\hline $\begin{array}{l}\text { Senecio genisianus } \\
\text { Cuatr. }\end{array}$ & $\begin{array}{l}\text { "Tutapure } \\
\text { Blanco" }\end{array}$ & Andes, Perú & $\begin{array}{l}\text { Heridas (limpieza), } \\
\text { Rabia, }\end{array}$ & & Infusión & $\begin{array}{l}\text { Hojas, } \\
\text { tallo }\end{array}$ & $\begin{array}{l}\text { Bussmann } \\
\text { \& Sharon } \\
2015\end{array}$ \\
\hline
\end{tabular}




\begin{tabular}{|c|c|c|c|c|c|c|c|}
\hline & & & $\begin{array}{l}\text { Mordeduras de } \\
\text { animales }\end{array}$ & & & & \\
\hline $\begin{array}{l}\text { Senecio } \\
\text { hypsiandinus } \\
\text { Cuatr. }\end{array}$ & $\begin{array}{l}\text { "Hórnamo } \\
\text { Blanco" }\end{array}$ & Andes, Perú & $\begin{array}{l}\text { Fragancia, Buena } \\
\text { Suerte }\end{array}$ & & Maceración & $\begin{array}{l}\text { Planta } \\
\text { entera, } \\
\text { tallo }\end{array}$ & $\begin{array}{l}\text { Bussmann } \\
\text { \& Sharon } \\
2015\end{array}$ \\
\hline $\begin{array}{l}\text { Senecio otuscensis } \\
\text { Cabrera }\end{array}$ & "Árnica" & Andes, Perú & $\begin{array}{l}\text { Inflamación, } \\
\text { Reumatismo, } \\
\text { Fiebre alto }\end{array}$ & & Decocción & $\begin{array}{l}\text { Hojas, } \\
\text { tallo }\end{array}$ & $\begin{array}{l}\text { Bussmann } \\
\text { \& Sharon } \\
2015\end{array}$ \\
\hline $\begin{array}{l}\text { Senecio } \\
\text { tephrosioides } \\
\text { Turcz. }\end{array}$ & $\begin{array}{l}\text { "Huamanripa", } \\
\text { "Genciana" }\end{array}$ & Andes, Perú & $\begin{array}{l}\text { Bronquitis, Asma, } \\
\text { Neumonía }\end{array}$ & & Infusión & $\begin{array}{l}\text { Planta } \\
\text { entera }\end{array}$ & $\begin{array}{l}\text { Bussmann } \\
\text { \& Sharon } \\
2015\end{array}$ \\
\hline $\begin{array}{l}\text { Senecio nutans } \\
\text { Sch. Bip }\end{array}$ & "Wiskataya" & $\begin{array}{l}\text { Puquio, } \\
\text { LucanasAyacucho }\end{array}$ & $\begin{array}{l}\text { Analgésico, } \\
\text { antitusígeno, } \\
\text { afecciones } \\
\text { respiratorias }\end{array}$ & $\begin{array}{l}\text { 1-isopropil- 4-metil biciclo } \\
{[3,1,0] \text { hexano y terpineno }}\end{array}$ & Infusión & $\begin{array}{l}\text { Parte } \\
\text { aérea }\end{array}$ & $\begin{array}{l}\text { Alderete } \\
2017\end{array}$ \\
\hline $\begin{array}{l}\text { Senecio calvus } \\
\text { Cuatrec }\end{array}$ & "Huamanripa" & $\begin{array}{l}\text { Huancayo, } \\
\text { Huancayo - Junín }\end{array}$ & $\begin{array}{l}\text { Antitusígeno, } \\
\text { antiflamatorio, } \\
\text { antimicrobiano, } \\
\text { analgésico }\end{array}$ & $\begin{array}{l}\text { 1-isopropil- 4-metil biciclo } \\
{[3,1,0] \text { hexano y terpineno }}\end{array}$ & Infusión & $\begin{array}{l}\text { Parte } \\
\text { aérea }\end{array}$ & $\begin{array}{l}\text { Alderete } \\
2017\end{array}$ \\
\hline $\begin{array}{l}\text { Senecio } \\
\text { chiquianensis } \\
\text { Cabrera }\end{array}$ & "Remilla" & $\begin{array}{l}\text { Caraz, Huaylas - } \\
\text { Ancash }\end{array}$ & $\begin{array}{l}\text { trastornos renales } \\
\text { y úlceras }\end{array}$ & $\begin{array}{l}\text { 1-isopropil- 4-metil biciclo } \\
{[3,1,0] \text { hexano y terpineno }}\end{array}$ & $\begin{array}{l}\text { Infusión, } \\
\text { decocción y } \\
\text { cataplasma }\end{array}$ & $\begin{array}{l}\text { Parte } \\
\text { aérea }\end{array}$ & $\begin{array}{l}\text { Alderete } \\
2017\end{array}$ \\
\hline $\begin{array}{l}\text { Senecio } \\
\text { coymolachensis } \\
\text { Cabrera }\end{array}$ & "Chinalinda" & Cajamarca & $\begin{array}{l}\text { Enfermedades de } \\
\text { la piel }\end{array}$ & & Decocción & Planta & Orillo 2018 \\
\hline $\begin{array}{l}\text { Senecio } \\
\text { pseudotites Griseb }\end{array}$ & "Árnica" & Cajamarca & $\begin{array}{l}\text { Sistema uro } \\
\text { genital }\end{array}$ & & Infusión & Hoja & Orillo 2018 \\
\hline $\begin{array}{l}\text { Senecio radiatus } \\
\text { Cuatrec. }\end{array}$ & $\begin{array}{l}\text { "Vira vira de } \\
\text { jalca" }\end{array}$ & Cajamarca & $\begin{array}{l}\text { Sistema } \\
\text { respiratorio }\end{array}$ & & Decocción & Hoja & Orillo 2018 \\
\hline $\begin{array}{l}\text { Senecio calvus } \\
\text { Cuatrecasas }\end{array}$ & $\begin{array}{l}\text { "huamanripa } \\
\text { serrana" }\end{array}$ & Lima & $\begin{array}{l}\text { Antiflamatorio, } \\
\text { asma } \\
\text { bronquial, tos } \\
\text { crónica, } \\
\text { bronconeumonía }\end{array}$ & $\begin{array}{l}\text { flavonoides, taninos y } \\
\text { terpenos }\end{array}$ & Infusión & $\begin{array}{l}\text { Hojas, } \\
\text { tallo }\end{array}$ & $\begin{array}{l}\text { Ajahuana } \\
2018\end{array}$ \\
\hline
\end{tabular}




\section{Senecio tephrosioides "Huamanripa":} información botánica y aplicaciones

Es una hierba perenne rizomatosa, de altura aproximada entre $25-40 \mathrm{~cm}$, el tallo es escapiforme, con hojas basales semiarrosetadas, y el peciolo desarrolla entre $3-5 \mathrm{~cm}$ de largo, violácea clara membranácea y glabra, limbo oblongo espatulado y oblongo lanceolado, 6-9 x 0,5- $2 \mathrm{~cm}$, margen revoluto de bordes enteros, ápice semiagudo, atenuado en la base, glabro en ambas caras, nervadura central notoria en el envés, hojas caulinares alternas, sésiles capítulos discoideos, en corimbos laxos; involucro discoide, caliculado 10-15 x 10-15mm, brácteas uniseriadas, lineal lanceoladas agudas. Pilosas en el ápice. Flores amarillas, actinomorfas, isomorfas, hermafroditas; corola tubulosa amarillenta, pentadentada; anteras concrescentes, ápice apendiculado, base obtusa, filamentos lacriformes. Aquenio cilíndrico y glabro. Pappus formados por números pelos blanquecinos (Fig. 1). En cuanto a su hábitat, vive en la puna entre gramíneas en zonas rocosas, florecen en abril y mayo, crece protegida entre rocas arriba de los 3000-4500m en los departamentos de Piura, Ancash, Cusco, Puno (Loja 2002).

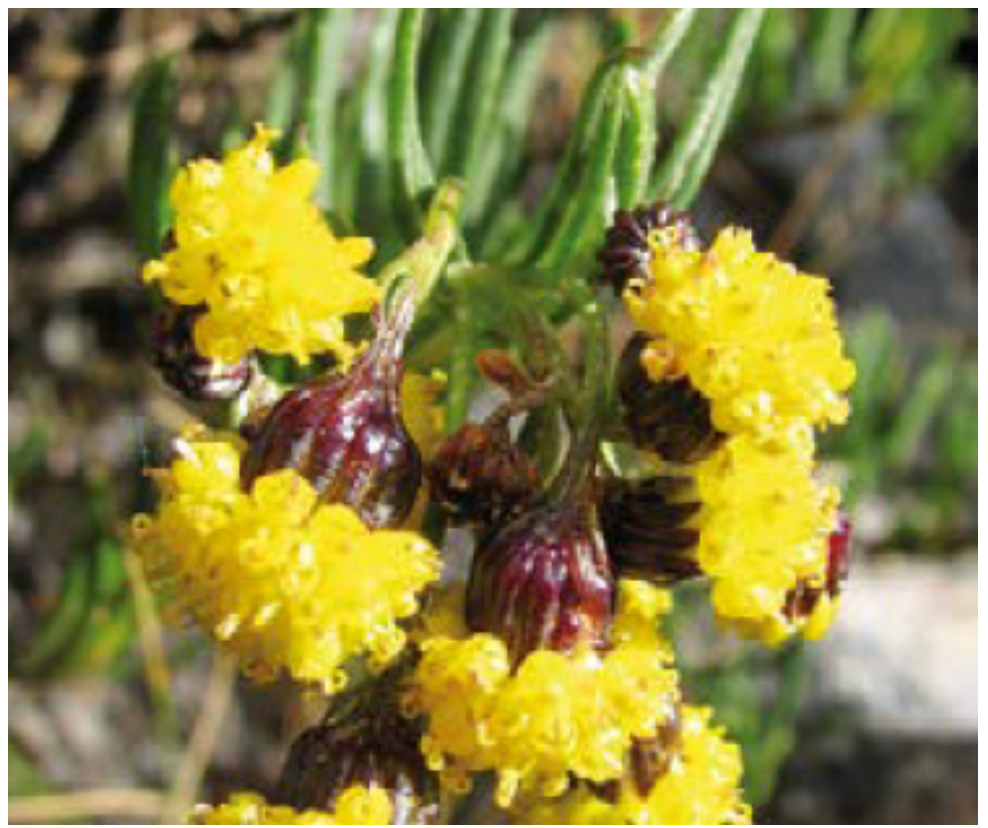

Figura 1. Senecio tephrosioides "huamanripa" (Photo: Bussmann \& Sharon 2015)

\section{Compuestos químicos bioactivos}

Las investigaciones sobre los componentes fitoquímicos de las especies de Senecio reportan una serie de clases de compuestos fisiológicamente activos, como terpenos, compuestos fenólicos, flavonoides, taninos, aceites esenciales, alcaloides y lactonas. Sin embargo, en Perú se han reportado pocos estudios fitoquímicos sobre este género. Alderete (2017) determinó la composición química y la actividad antimicrobiana de los aceites esenciales de tres especies de Senecio, entre ellas "huamanripa", por análisis de cromatografía de gases (CG/EM) se reveló que los hidrocarburos monoterpenos fueron los componentes mayoritarios, seguidos de los sesquiterpenos, específicamente se elucidaron componentes como 1-isopropil- 4-metil biciclo $[3,1,0]$ hexano y terpineno (fig. 2).

Soriano junto a otros investigadores (2004), realizaron un estudio fitoquímico de Senecio calvus en el departamento de Lima, donde determinaron que el extracto etanólico contiene flavonoides y taninos en mayor proporción, en menor proporción se encontró alcaloides y terpenos. el objetivo principal de la presente investigación fue elucidar la estructura química de algunos metabolitos secundarios presentes en las hojas, así como evaluar su actividad cicatrizante. La elucidación estructural se realizó mediante espectroscopia ultravioleta-visible y reacciones químicas, determinando que los compuestos fitoquímicos de esta especie pertenecían a compuestos fenólicos tipo flavonoides-derivados del núcleo flavanonona (5,7-dihidroxi-4',6',8-trimetoxiflavanonona,3',5'-

dihidroxi-4-metoxi-7-0-rhannoglucosyl flavanonona) y una chalcona (5,4-dihidroxichalcona). (fig. 2) A pesar de la información existente, los estudios fitoquímicos realizados en Perú, sobre las diversas especies de este género a la fecha son escasos, comparando la cantidad de especies que existen en el país y que aún no han sido estudiadas más allá de su uso tradicional, por lo que se sugiere que se realicen más investigaciones sobre los compuestos 
ya reportados de Senecio para dilucidar sus actividades farmacológicas a fondo, ya que antecedentes etnobotánicos indican que este género es ampliamente utilizado de forma tradicional

Flavonoides del género Senecio<smiles>[R][R]Cc1c([R])c([R])c2c(c1[R])OC(c1ccc([R])c([R])c1)CC2=O</smiles>

(5,7-dihidroxi-4',6', 8-trimetoxiflavanonona), $4^{\prime}, 6,8=\mathrm{R}=\mathrm{OCH} 3 ; 5,7=\mathrm{R}^{\prime}=\mathrm{OH}$ en diversas comunidades del Perú como Piura, Anchash, Puno, Cuzco, con mayor relevancia en las zonas altoandinas del país (Mostacero et al. 2011).<smiles>O=C(/C=C/c1ccc(O)c(O)c1)c1ccccc1</smiles>

5,4-dihidroxichalcona<smiles>O=c1c(O[C@@H]2O[C@H](O)[C@@H](O)[C@H](O)O2)c(-c2ccc(O)c(O)c2)oc2cc(O)cc(O)c12</smiles>

Rutina (quercetin-3-rutinósido)

Terpenos de género Senecio<smiles>CC(C)C12CCC(CC1C)C2C</smiles>

1-isopropil- 4-metil biciclo $[3,1,0]$ hexano<smiles>CC1CCC(C(C)C)CC1</smiles>

Terpineno

Figura 2. Estructuras químicas de compuestos bioactivos del género Senecio

Actualmente el Centro de medicina complementaria de EsSalud, también viene trabajando estas especies, por lo que recalca nuevamente la importancia de realizar investigaciones más completas en esta área, si bien la información etnobotánica es importante, y es una base importante para continuar con la parte de estudios fitoquímicos y farmacológicos, que dar un valor científico al conocimiento tradicional, protegiendo la salud e integridad de los consumidores y no solo eso, sino que el estudio de estas especies de plantas ayuda a ampliar el alcance para el desarrollo de candidatos para nuevas drogas 0 bioformulaciones en el tratamiento de enfermedades, respectivamente. 
Sin embargo, en américa latina existen diversos reportes de estudios en los que trabajaron con diferentes especies de Senecio, tal es el caso de Senecio scandens Buch.-Ham., de esta especie se han aislado e identificado alrededor de 180 compuestos de entre ellos flavonoides, por ejemplo se aisló hiperósido y linarina de toda la planta medicinal de Senecio scandens, así mismo, se aislaron e identificaron cuatro flavonoides: isoquercitrina, hiperina y quercetina a partir del extracto de acetato de etilo de Senecio scandens, del extracto acuoso de Senecio scandens se determinó alcaloides pirrolizidínicos: neoplatifilina, Senecionina, seneciphylline, seneciphylline $\mathrm{N}$-óxido y senkirkine (Fig. 3), los cuales son hepatotóxicos y tumorigénicos. Los metabolitos se analizaron mediante cromatografía líquida de ultra rendimiento (UPLC)/espectrometría de masas por ionización por electropulverización (Wang 2013).

\section{Alcaloides pirrolizidínicos}<smiles>C/C=C(/CC(C)[C@@H](C)O)C(=O)O[C@H]1CCN2CC[C@H](COC)C12</smiles>

Neoplatifilina<smiles>C/C=C(/C[C@@H](C)[C@@](C)(O)C(=O)OCC1=CCN2CC[C@H](OC1=O)[C@H]2C)C(=O)O</smiles>

Senecionina<smiles>C=C(CO)C/C(=C/C)C(=O)O[C@H]1CCN2CC=C(COC(=O)[C@](C)(O)C(=O)O)[C@H]12</smiles>

Seneciofilina<smiles></smiles>

Senecionina $\mathrm{N}$-oxido<smiles>C/C=C1/C[C@@H](C)[C@@](C)(O)C(=O)OCC2=CCN(C)CC[C@@H](OC(=O)C2=O)C1=O</smiles>

Serkinkine

Figura 3. Alcaloides pirrolizidínicos presentes en diferentes especies del género Senecio

Alderete (2017), evaluó Actividad antimicrobiana, antioxidante in vitro y composición química de los aceites esenciales de Senecio nutans Sch. Bip "Wiskataya", Senecio calvus Cuatrec "Huamanripa" y Senecio chiquianensis Cabrera "Remilla" procedentes de Ayacucho, Huancayo y Ancash, las muestras fueron sometidos a la determinación de la composición química mediante Cromatografía de Gases /Espectrometría de Masas (CG/EM), donde se reveló que los hidrocarburos monoterpenos fueron los componentes mayoritarios, seguidos de los sesquiterpenos. Así mismo dentro de la determinación de la composición química de los tres aceites esenciales, se elucidaron componentes frecuentes, 1-isopropil- 4-metil biciclo $[3,1,0]$ hexano y terpineno (Fig. 2).

\section{Usos tradicionales}

El género Senecio agrupa una gran cantidad de especies, que, de manera similar son utilizadas tradicionalmente para tratar diversas enfermedades (Fig. 4). Su uso es más frecuente en enfermedades respiratorias como, bronquitis, asma, neumonía (Bussmann \& Sharon 2015). Sin embargo, plantas de este género son utilizadas también como antinflamatorios, antimicrobianos y analgésicos (Deuschle et al. 2006). De acuerdo a los fitoconsituyentes encotrados en diversas especies del género Senecio, los compuestos terpenoides, están asociados a una amplia gama de las propiedades biológicas, como quimiopreventivos del cáncer, antioxidantes, antimicrobianas, antimicóticas, antivirales, antiinflamatorias y antiparasitarias (Alderete 2017).

Alderete en el 2017 reporta la capacidad antioxidante de los terpenos presentes en tres especies de Senecio, relacionando los resultados obtenidos con la literatura, donde refiere que la razón de la potencial actividad antioxidante se debe 
a la presencia de terpenos, particularmente aquellos con grupos metileno en sus moléculas, como se había mencionado anteriormente, los compuestos terpenicos elucidados fueron: 1-isopropil- 4-metil biciclo $[3,1,0]$ hexano y terpineno.

Entre los compuestos reportados de género Senecio también se encuentra la rutina (quercetin-3rutinósido), es un glucósido flavonoide el cual está relacionado con la actividad farmacológica de Senecio, principalmente la actividad antinflamatoria y antioxidante, se sabe que los flavonoides evitan la liberación de prostaglandinas, histaminas, que están relacionados con la cascada de la inflamación, además estabilizan la membrana celular capturando a los radicales libres y evitando daño celular, activando el sistema de complejo bioquímico para la regeneración del tejido (Soriano et al. 2004).

Pauro et al. (2011), realizaron estudio etnobotánico en la provincia de Lampa- Puno, tuvieron como objetivo determinar la cantidad de especies existentes en dicha comunidad, así como determinar su uso medicinal tradicionalmente usado, para ello aplicaron entrevistas a representantes de las familias de las comunidades, reportando 214 especies, 140 géneros y 52 familia; donde la familia con mayor predominancia es Asteracecae. Así mismo dentro de este estudio, se puede evidenciar en el reporte, que hay diferentes especies del género Senecio (Senecio tephrosioides Turcs, Senecio graveolens Wedd., Senecio sp., Senecio canescens (H. \& B. Cuatr.), reportando también las aplicaciones de estos en la esta comunidad, no solo como agente medicinal (antitusígeno, bronquitis, antiflamatorio), sino también son utilizadas como plantas biocidas, aprovechando su efecto en casos de plagas y enfermedades de cultivos por sus propiedades repelentes.

En el 2018, Ajahuana realizó un estudio en la Universidad Inca Garcilaso de la Vega, donde evaluaron el efecto antiinflamatorio del extracto etanólico de las hojas y tallos del Senecio calvus Cuatrecasas (huamanripa serrana) en ratas con edema plantar inducidas con carragenina, donde obtuvieron buenos resultados, ya que la especie presentó efecto antiinflamatorio a concentración de $500 \mathrm{mg} / \mathrm{kg}$ en (38.99\%), previo al estudio farmacológico realizó una marcha fitoquímica preliminar para determinar los metabolitos presentes en la especie, encontrando alcaloides, compuestos fenólicos: flavonoides, taninos y terpenos. Así mismo realizaron un ensayo comparativo donde determinaron que el extracto etanólico del Senecio calvus presento mayor porcentaje del efecto antinflamatorio a concentración $500 \mathrm{mg} / \mathrm{kg}$ en comparación con el fármaco ácido acetil salicílico en $24.70 \%$ pero en menor grado que el naproxeno sódico (43.75\%), el efecto antiflamatorio que presenta "huamanripa" está relacionado a los flavonoides presentes en esta planta, se sabe que los flavonoides evitan la liberación de prostaglandinas, histaminas, evitan la migración neutrófilos y otros mediadores que están relacionados con la cascada de la inflamación.

Hammond et al (1998), realizaron un estudio etnobotánico en el Callejón de Huaylas, Departmento de Ancash, donde informan los usos tradicionales de diversas especies en la comunidad, destacando el uso tradicional de Senecio tephrosioides Turcz. (IFV227), hierba silvestre utilizada para tratar síntomas de tos, y en situaciones patológicas de Bronquitis, como se había mencionado anteriormente, el género Senecio se caracteriza por poseer constituyentes como flavonoides, los cuales además de estar relacionados con la actividad antiflamatoria, también tienen actividad sobre bacterias (procesos infecciosos), su actividad sobre las bacterias probablemente se deba a su capacidad de generar complejos con proteínas extracelulares y proteínas solubles, así como una actividad sobre la pared celular, muy similar a la de las quinonas (Soto 2015).

Kahriman et al (2011), evaluaron la composición química y activida antimicrobiana de los aceites esenciales de la flores, hojas y tallo de Senecio pandurifolius, reportando que el perfil químico revela el predominio de los hidrocarburos sesquiterpénicos (flor: $42.4 \%$, hoja: $43.4 \%$, tallo: $52.3 \%$ ). Los componentes principales de los aceites esenciales propios de $S$. pandurifolius fueron $\alpha$-cuprenene (30.7\%) en flor, $\alpha$-zingiberene (16.1\%) en hoja y $\gamma$ curcumene $(14.9 \%)$ en tallo. En cuanto a las actividades antimicrobianas mostraron actividad contra bacterias Gram positivas, micobacterias y hongos, pero no bacterias Gram negativas. Concluyendo que el aceite esencial extraido de la hoja mostro alta actividad antimico-bacteriana en comparación con los otros, sugiriendo que los resultados obtenidos merecen más investigación para determinar sus componentes activos. Así mismo indican de manera muy similar a otros estudios, el uso de especies de Senecio para el tratamiento del asma, la tos, la bronquitis, el eccema y la cicatrización de heridas.

Los terpenos son activos contra bacterias, hongos y virus. Tienen generalmente acción depresora sobre la tensión superficial lo cual, cuando tiene lugar en el entorno de la célula bacteriana, altera la selectividad de la membrana citoplasmática para el intercambio de sustancias. Así mismo, los triterpenos de naturaleza alcohólica pueden alterar 
la naturaleza coloidal del protoplasma de la célula provocando su muerte (Soto 2015).

Florián (2014) evaluó los Principios activos de Senecio calvus en la Formación de biopelículas de contra Pseudomonas aeruginosa", bacteria oportunista muy utilizada debido a que es una bacteria que forma rápidamente biopelículas. Se mezclaron los cultivos de $P$. aeruginosa en fase de crecimiento junto a los extractos y fracciones de $\mathrm{S}$. calvus para determinar el grado de inhibición de las biopelículas; para tal efecto se usaron concentraciones subinhibitorias. Los resultados arrojaron una inhibición de 92.9 y $76.4 \%$ en dos de los extractos y de hasta $88 \%$ en las fracciones cromatograficas, lo cual indica que Senecio calvus es un buen candidato para el aislamiento de una molécula inhibidora de biopelículas con potencial patentable.

Las plantas medicinales suelen ser preparadas y aplicadas de diversas maneras, y este es un factor muy importante a tener en cuenta con relación al éxito del tratamiento tradicional. Cada país, región, comunidad, adoptan un sistema de curación que puede ser igual o diferente a la otra, estos distintos modos de aplicación son utilizados en base a la experiencia, tal es el caso de las flores y hojas que son preparadas tradicionalmente por el método de infusión que consiste en añadir el material vegetal en agua a $100{ }^{\circ} \mathrm{C}$, y dejar en contacto a temperatura ambiente (Malca et al. 2019, Handa et al. 2008), ya que, son órganos más sensibles al calor y al entrar en contacto con este van a liberar más fácilmente el principio activo, mientras, en el caso de cortezas y raíces, la forma tradicionalmente de preparar es por decocción, debido a la naturaleza de estos, ya que tienden a ser órganos más robustos, más duros y por ende necesitan de un método más eficiente al momento de extraer sus principios activos. En el caso de Senecio tephrosioides "huamanripa", junto a otras especies del mismo género, la forma de preparación más común es por el método de infusión (Bussmann \& Sharon 2015, Mostacero et al. 2011), ya que la parte más utilizada de esta planta suelen ser las hojas.

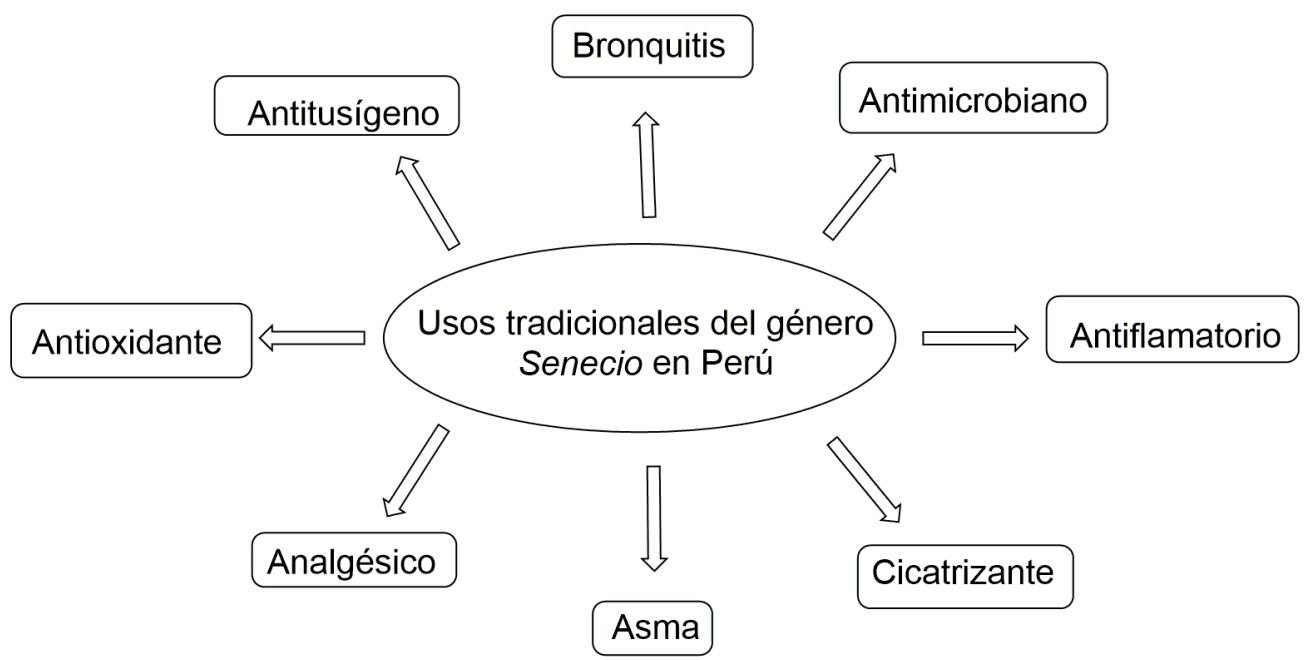

Figura 4. Usos tradicionales del género Senecio en Perú

\begin{abstract}
¿Existen otras especies Ilamadas bajo el mismo nombre vulgar de Huamanripa?

Reporte de especies del genero Senecio llamadas bajo el mismo nombre vulgar

Durante la búsqueda bibliográfica, se pudo encontrar que Senecio tephrosioides no es la única especie llamada bajo el mismo nombre vulgar "huamanripa", en un estudio publicado por Beltrán y Roque en el 2015, indican que la especie Senecio violaefolius Cabrera, es Ilamada comúnmente como "huamanripa" y "wamanlipa" en las Altas montañas desde el centro del Perú hasta Bolivia.
\end{abstract}

Por otro lado, también se encontró que Senecio arachnolomus (Wedd.) Chiov. También es conocida comúnmente como "huamanripa", la especie fue colectada en Anchash donde mediante entrevistas etnobotánicas obtuvieron información del nombre vulgar. Asi mismo la distribución de la especie es endémica del Perú, y es usada como medicinal de manera similar a las otras especies del genero Senecio. (Beltrán \& Roque 2015). Senecio calvus Cuatrecasas, también es llamado por el nombre vulgar de "huamanripa". Los pobladores de Ancash refieren suplir sus dolencias mediante el uso de estas plantas debido a sus propiedades antiflamatorias, estos efectos son relacionados a los fitoconstituyentes que tiene la planta como, flavonoides y alcaloides.

El problema de que se llame a diferentes especies por el mismo nombre vulgar, y peor aún, estas sean 
comercializadas en los mercados, es un peligro para la integridad de la población, ya que estas personas están consumiendo algo que hasta el propio vendedor desconoce información, por ejemplo, si la planta funciona y lo más importante si su consumo es seguro. Estos sucesos hacen que se piense que aun actuando de manera primitiva, porque seguimos experimentando, al permitir que exista el comercio y consumo "a ciegas" de plantas "con propiedades medicinales" sin estar científicamente identificadas y validadas, con ello, no se pretende desprestigiar el gran valor y propiedad de algunas plantas, pero si es importante dar mayor énfasis en la parte investigativa de esta área, para evitar daños futuros, ya que hay plantas que son fuente de metabolitos altamente tóxicos y perjudiciales para la salud.

Existen más de una especie de Senecio llamado bajo el mismo nombre vulgar "huamanripa", sin embargo, lo que no existe son registros de estudios fitoquímicos, farmacológicos de todas las especies que respalden su valor tradicional para la cual son utilizados, en la presente revisión se han encontrado 5 especies de Senecio llamadas bajo el mismo nombre vulgar, de las cuales solo 2 especies tienen registro de estudios fitoquimicos y biológicos, como se puede apreciar en la Tabla 1.

Así mismo, se puede apreciar que en los últimos años, la aceptación de las terapias tradicionales por parte de las comunidades ha crecido, así como la demanda de los mismos, esto genera preocupación principalmente en aquellas plantas medicinales que son nativas y que corren riesgo de extinción, tal es el caso de las diferentes especies de Senecio identificadas en el país, todas ellas nativas, sin embargo no existe reporte de estudios de adulteración de plantas, información que sería importante conocer, porque, el que una planta entre en riesgo de extinción significa que pueden ser fácilmente reemplazadas por los comerciantes por otras plantas que tengan las mismas características físicas, como sucedió en Bolivia, con la especie Equisetum giganteum "cola de caballo" utilizada para problemas urinarios e inflamaciones renales que es reemplazada por Ephedra americana Humb., la cual es vendida también bajo el mismo nombre "cola de caballo", e indicada exactamente para lo mismo (Macía et al. 2005), esto hace ver la importancia de realizar estudios no solo de laboratorios sino también de mercado y valorar la realidad que se vive en diversas comunidades del país, ya que situaciones como el caso anterior de reemplazo de especies, ponen en riesgo la seguridad y salud de las personas.

Otro punto importante al que se le debe dar interés es a los alcaloides tóxicos que presentan diferentes especies del genero Senecio, del cual tampoco hay reporte de estudios en Perú, pero si en otros países, en donde se han encontrado alcaloides pirrolizidinicos que son conocidos por sus propiedades hepatotóxicas (Wang et al 2013).

\section{Conclusiónes}

Esta revisión resumió las características etnobotánicas, metabolitos secundarios principales, etnofarmacología y posibles usos medicinales de Senecio tephrosioides. Los estudios fitoquímicos, biológicos de eficacia y toxicidad de Senecio tephrosioides necesitan ser más explorados y discutidos. La caracterización de plantas usadas tradicionalmente es una gran controversia, por lo tanto, los metabolitos secundarios se deben identificar y caracterizar analíticamente, mediante métodos ortogonales como Resonancia Magnética Nuclear (RMN), Cromatografía Liquida de Alta Resolución (HPLC) y Espectrometría de Masas acoplado a Cromatografía Liquida (MS-LC), estas técnicas no solo permiten identificar grupos de metabolitos presentes sino permite elucidar la estructura química de dichos compuestos químicos responsables de la actividad biológica de los mismos. Dado que actualmente se viene trabajando esta especie junto a otras especies de plantas medicinales en el Centro de Medicina Complementaria-Essalud, es importante realizar estudios que validen experimentalmente las posibles interacciones entre medicamentos y extracto de plantas, ya que en determinados casos estas son utilizadas paralelamente.

\section{Recomendación}

Los estudios fitoquímicos deberían dar mayor importancia a todas las plantas utilizadas en la medicina tradicional (en general), y no solo enfocarse en el estudio de algunas especies que están "de moda" o que son comercializadas en escala internacional. Finalmente se debería hacer un compendio de plantas medicinales científicamente validadas en Perú, con ello existiría constancia de que estas plantas funcionan y son seguras, así como también, aprovechar la información que ya existe en diferentes bases de datos online como Journal of Ethnopharmacology, Ethnobotany Research and Applications, donde se puede encontrar información diversa de estudios etnobotánicos y etnofarmacológicos, aprovechando los métodos y técnicas empleadas en dichos estudios como herramientas para futuras investigaciones.

\section{Declaraciones}

Lista de Abreviaturas: RMN: Resonancia Magnética Nuclear; HPLC: Cromatografía Líquida 
de Alta Resolución; MS-LC: Espectrometría de Masas - acoplado a Cromatografía Líquida; CG: cromatografía de gases; UPLC: cromatografía líquida de ultra rendimiento; MT: Medicina Tradicional; MAC: Medicina AlternativaComplementaria; OMS: Organización Mundial; OPS: Organización Panamericana de la Salud; MC: Medicina complementaria; AP: alcaloides de pirrolizidina; HUT: Herbarium truxillense; HMHN: Herbario Museo de Historia Natural; MOL: Herbario Weberbauer de la Universidad Nacional Agraria La Molina; CUZ: Herbario Vargas Cuz.

Aprobación ética y consentimiento para participar: No Aplica

Consentimiento para la publicación: No aplica

Disponibilidad de datos y materiales: $\mathrm{Si}$ hay conjuntos de datos depositados en repositorios públicos

Conflicto de intereses: Los autores declaran no tener conflicto de interés relacionados con el tema descrito en esta revisión.

Financiación: Esta revisión fue realizada dentro del Programa de Doctorado en Farmacia y Bioquímica de la Universidad Nacional de Trujillo. Perú financiado por el Consejo Nacional de Ciencia y Tecnología - Perú (CONCYTEC) en cooperación con Banco Mundial (Contrato N 07-2018FONDECYT-BM-IADT-MU).

Contribuciones de los autores: Blanco-Olano C.: búsqueda y análisis de la información y redacción del manuscrito, elaboración de estructuras y tablas. Olascuaga-Castillo K.: elaboración de esquemas y estructuras químicas de y análisis de la etnofarmacología. Rubio-Guevara S.: elaboración de búsqueda de información etnobotánica y análisis de relación con compuestos químicos. ValdiviezoCampos JE.: Elaboración de estructuras químicas, análisis y redacción de la parte fitoquímica.

\section{Literatura citada}

Ajahuana TM. 2018. Efecto antiinflamatorio del extracto etanólico de las hojas y tallos del Senecio calvus Cuatrecasas "huamanripa serrana" en ratas con edema plantar inducidas con carragenina. Tesis de Grado. Universidad Inca Garcilazo de la Vega. Lima - Perú. 13-90

Alderete H. 2017. Actividad antimicrobiana, antioxidante in vitro y determinación de la composición química de tres aceites esenciales del género Senecio del Perú. Tesis grado. Universidad Nacional Mayor de San Marcos. Lima- Perú. 10-60

Alzamora L, Morales L, Armas L, Fernández G. 2001. Medicina Tradicional en el Perú: Actividad Antimicrobiana in vitro de los Aceites Esenciales Extraídos de Algunas Plantas Aromáticas. Anales de la Facultad de Medicina. Universidad Nacional Mayor de San Marcos 62(2)156-161.
Beltrán H, Granda A, León B, Sagástegui A, Sánchez I, Zapata M. 2006. Asteráceas endémicas del Perú. Revista Peruana de Biología 13(2):64-164.

Beltrán H, Granda A. 2017. Nuevas especies de Senecio (Asteraceae, Senecioneae) del sur del Perú. Revista peruana de biología 24(2): 205-210

Beltrán H, Roque J. 2015. El género Senecio L. (Asteraceae-Senecioneae) en el departamento de Lima, Perú. Arnaldoa 22(2):395-412

Beltrán H. 2016. Las Asteráceas (Compositae) del distrito de Laraos (Yauyos, Lima, Perú). Revista peruana de biología 23(2):195-220

Brako L, Zarucchi J. 1993. Catalogue of the Flowering Plants and Gymnosperms of Peru. Monographs in Systematic Botany from the Missouri Botanical Garden 45:1-1286.

Bussmann RW, Sharon D, Vandebroek I, Jones A, Revene Z. 2007. Health for sale: the medicinal plant markets in Trujillo and Chiclayo, Northern Peru. Journal of Ethnobiology and Ethnomedicine 3:37-38.

Bussmann RW, Glenn A, Sharon D. 2010. Antibacterial activity of medicinal plants of Northern Peru - can traditional applications provide leads for modern science? Indian Journal of Traditional Knowledge 9(4)742-753.

Bussmann RW, Malca G, Glenn A, Sharona D, Nilsen B, Parris B, Dubose D, Ruiz D, Saleda J, Martinez M, Carillo L, Walker K, Kuhlman A, Townesmith A. 2011. Toxicity of medicinal plants used in traditional medicine in Northern Peru. Journal of Ethnopharmacology 137:121- 140.

Bussmann RW, Sharon D. 2016. Plantas medicinales de los Andes y la Amazonía-La flora mágica y medicinal del Norte del Perú. Ethnobotany Research and Applications 15(1):42,43,108.

Cabrera A. 1957. El género Senecio (Compositae) en Brasil, Paraguay y Uruguay. Arquivos do Jardim Botânico do Rio de Janeiro 15:163-264.

Da Silva CM, Bolzan AA, Heinzmann BM. 2006. Alcalóides pirrolizidínicos em espécies do gênero Senecio. Química Nova 29(5)1047-1053.

De La Torre L, Muriel P, Balslev H. 2006. Etnobotánica en los Andes del Ecuador. Revista Botánica Económica de los Andes Centrales. Universidad Mayor de San Andrés, La Paz, Quito Ecuador. 247:246-267

Deuschle R, Camargo T, Francescato L, Laves S, Heinzmann B. 2006. Antimicrobial activity of Senecio desiderabilis Vellozo (Asteraceae). Acta Farmacéutica Bonaerense 25(3):356-359.

Florian JC. 2014. Evaluación de los principios activos de Senecio calvus en la formación de biopelículas de Pseudomona aeruginosa. Tesis de grado. Universidad Nacional Mayor de San Marcos. Lima - Perú. 22-49 
Habermehl GG, Martz W, Tokarnia CH, Döbereiner J, Mendez MC. 1988. Livestock poisoning in south america by species of the Senecio plant. Toxicon 26(3)275-286

Hammond G, Fernandez I, Villegas L, Vaisberg A. 1998. A survey of traditional medicinal plants from the Callejón de Huaylas, Department of Ancash, Peru. Journal of Ethnopharmacology 61:17-30.

Handa SS, Khanuja SP, Longo G, Rakesh DD. 2008. Extraction Technologies for Medicinal and Aromatic Plants. Earth, environmental and marine sciences and technologies, Trieste, Italia.62-81

IUCN Standards and Petitions Subcommittee. 2014. Guidelines for using the IUCN Red List categories and criteria. Version 11. Prepared by the Standards and Petitions Subcommittee.

Mejía JA, Carrasco E, Miguel JL, Flores SA. 2017. Conocimiento, aceptación y uso de medicina tradicional peruana y de medicina alternativa/complementaria en usuarios de consulta externa en Lima Metropolitana. Revista Peruana de Medicina Integrativa 2(1):47-57.

Kahriman N, Tosun G, Terzioğlu S, Alpay Ş, Yaylı N. 2011. Chemical Composition and Antimicrobial Activity of the Essential Oils from the Flower, Leaf, and Stem of Senecio pandurifolius, Records of Natural Products 5(2):82-91.

Loja HB. 2002. Contribución al estudio florístico de la provincia de Concepción, Junín: Dicotiledóneas. Tesis postgrado. Universidad Mayor de San Marcos. Lima - Perú. 11,98-101

López MG, Xifreda CC, Poggio L y Wulff AF. 2013. Deep cytogenetics analysis reveals meiotic recombination depletion in species of Senecio (Asteraceae). Botanical Studies 54(1):20

Montes B. 2017. Estudios exomorfológicos, anatómicos y palinológicos en el género Senecio L. (Asteraceae): comparación de especies pertenecientes a las series XeroSenecio, Corymbosi, Culcitium y Otopteri. Tesis postgrado. Universidad Nacional del Sur. Bahía Blanca Argentina.

Macía MJ, García E, Vidaurre PJ. 2005. An ethnobotanical survey in the markets of La Paz and El Alto, Bolivia. Journal of Ethnopharmacology 97:337-350.

Malca-Garcia GR, Zagala D, Grahamb J, Nikolića D, Friesen JB, Lankina DC, Shao-Nong C, Paulia GF. 2019. Dynamics of the isoflavone metabolome of traditional preparations of Trifolium pratense L. Journal of Ethnopharmacology 238:1-12

Milton JJ. 2009. Phylogenetic analyses and taxonomic studies of Senecioninae: southern African Senecio section Senecio. Thesis of PhD. University of St. Andrews. United Kingdom. 14-182
Montesinos DB, Gonzáles P, Navarro E. 2015. Senecio canoi (Compositae), una especie nueva de los Andes de Perú. Anales del Jardín Botánico de Madrid 72(2)1-4

Mostacero J, Castillo F, Mejía FR, Gamarra OA, Charcape JM, Ramírez RA. 2011. Plantas medicinales del Perú. Taxonomía, Ecogeografía, Fenología y Etnobotánica.Editorial ANR. Lima, Perú. 562

Nordenstam B, Pelser P, Kadereit J, Watson L. 2009. Senecioneae. In: Funk, V.A., Susanna, A., Stuessy, T.F. \& Bayer, R.J. Systematics, Evolution and Biogeography of Compositae. International Association for Plant Taxonomy, Institute of Botany, University of Vienna. 503-521.

Organización Panamericana de la Salud. Situación de las plantas medicinales en Perú. Informe de reunión del grupo de expertos en plantas medicinales. (Lima, 19 de marzo del 2018). Lima: OPS; 2019.

Orrillo R. 2018. Etnobotánica de las plantas medicinales expendidas en los mercados de Cajamarca y San Marcos. Tesis de grado. Universidad Nacional de Cajamarca. CajamarcaPerú. 1-64

Pauro R, Gonzáles M, Gamarra C, Pauro R, Mamani M.; Huerta, R. 2011. Plantas alimenticias, medicinales y biocidas de las comunidades de Muñani y Suatia, provincia de Lampa (Puno - Perú). Ecología Aplicada 10(1):41-49.

Pelser PB, Nordenstam B, Kadereit JW, Watson LE. 2007. An ITS phylogeny of tribe Senecioneae (Asteraceae) and a new delimitation of Senecio L. Taxon 56(4):1077-1104.

Bussmann RW, Malca G, Glenn A, Sharon D, Nilsen B, Parris B, Dubose D, Ruiz D, Saleda J, Martinez M, Carillo L, Walker K, Kuhlman A, Townesmith A. 2011. Toxicity of medicinal plants used in traditional medicine in Northern Peru. Journal of Ethnopharmacology 137:121-140

Rojas R, Bustamante B, Bauer J, Fernández I, Albán J, Lock, O. 2003. Antimicrobial activity of selected Peruvian medicinal plants. Journal of Ethnopharmacology 88(2-3)199-204.

Shi J, Yang L, Wang C, Chou G, Wang Z. 2007. A new lactone from Senecio scandens. Biochemical Systematics and Ecology 35(12):901-904.

Soriano M, Bonilla P, Arroyo J, Pereyra S. 2004. Actividad cicatrizante tópica de los metabolitos secundarios en el extracto etanólico de hojas de Senecio culcitoides Weed. Folia dermatológica peruana 15(3):155-159.

Vega E., López E. 2013. Concentración Mínima Inhibitoria del extracto hidroalcóholico de tallos y hojas de Baccharis genistelloides, Perezia multiflora, Senecio sublutescens y Jungia paniculata del 
Parque Nacional Huascarán (Perú) frente a cepas bacterianas de interés clínico. Revista Científica de Estudiantes 1(2):43-49.

Vision T, Dillon M. Sinopsis de Senecio L. 1996. (Senecionae, Asteraceae) para el Perú. Arnaldoa 4(1):23-46.

Wang D, Huang L, Chen S. 2013. Senecio scandens Buch.-Ham.: A review on its ethnopharmacology, phytochemistry, pharmacology, and toxicity. Journal of Ethnopharmacology 149(1):1-23

Yarupaitán G.; Albán J. 2004. Fanerógamas de la provincia de Huancayo, Perú. Revista Peruana de Biología 11(2):193-202. 